\title{
BER-Constrained Loading Algorithms for Multicarrier Spatial Diversity Systems
}

\author{
Alexander M. Wyglinski* Peter Kabal Fabrice Labeau \\ Centre for Advanced Systems and Technologies in Communications (SYTACom) \\ Department of Electrical and Computer Engineering, McGill University \\ 3480 University Street, Montreal, QC, Canada H3A 2A7
}

\begin{abstract}
In this paper, four novel loading algorithms are proposed for multicarrier systems employing spatial diversity. The objective of these algorithms is to increase system throughput while ensuring the mean bit error rate (BER) is below a specified limit. A subcarrier signal is transmitted simultaneously on a subset of antennas, passed through a multiple-input multiple output (MIMO) channel, intercepted by a subset of receive antennas, and linearly combined. Both antenna subset configurations may differ for each subcarrier. The rationale for this form of antenna selection is to efficiently distribute power across the antennas and to reduce array processing complexity. Bit loading is employed by two of the proposed algorithms to further increase throughput. Simulation results show a tradeoff between the allocation algorithm computational complexity and system throughput.
\end{abstract}

Keywords: Multicarrier Modulation, Bit Loading, Spatial Diversity, Antenna Selection

\section{Introduction}

Multiple antennas can be employed at the transmitter and receiver to enhance the error robustness of the system [1]. By transmitting multiple copies of the same signal, the likelihood that all of them would be severely corrupted by the channel decreases. This technique of transmitting the same signal across several antennas to mitigate poor channel conditions is known as spatial diversity. At the same time, when the channel paths between the transmit and receive antennas experience frequency-selective fading, multicarrier modulation can be employed by the system to transform these channels into a collection of approximately frequency-flat subchannels. As a result, this yields a lower implementation and computational complexity at the receiver with respect to equalization and detection [1].

Although system performance is improved when employing multiple antennas, there are also drawbacks, including increased power consumption and increased complexity of the array processing algorithms. One possible solution is antenna subset selection, also known as hybrid selection, where the

This work was partially supported by the Natural Science and Engineering Research Council of Canada and Le Fonds "Nature et Technologies" du Québec (NATEQ).

*A. M. Wyglinski is now at the Information and Telecommunication Technology Center, The University of Kansas, Lawrence, KS, USA 66045-7612. system chooses the best $L_{T}$ (resp. $L_{R}$ ) out of $N_{T}$ (resp. $N_{R}$ ) transmit (resp. receive) antennas. The values for $L_{T}$ and $L_{R}$ are fixed throughout the selection process. Many researchers have studied the use of antenna subset selection in both single carrier [2-6] and multicarrier systems [7-10].

Nevertheless, there exists the potential to further enhance system performance using antenna subset selection, at the cost of increased hardware complexity. First, conventional antenna subset selection algorithms fix the size of $L_{T}$ and $L_{R}$ by constraining the number of RF chains to be fewer than the number of antennas. However, this may result in an inefficient allocation of antennas. Second, none of the multicarrier systems employing antenna subset selection perform bit loading, a powerful technique to redistribute the data across the subcarriers to increase throughput while satisfying some error constraint [11]. Third, none of the algorithms perform antenna subset selection simultaneously at both the transmitter and the receiver independently for each subcarrier.

In this paper, we address these issues by proposing four novel allocation algorithms that activate specific antennas, or subcarriers per antenna, using an adapted version of antenna subset selection. Employing the proposed algorithms could potentially result in a reduction in both power consumption and array processing complexity. Unlike conventional antenna subset selection, the size of $L_{T}$ and $L_{R}$ are not restricted to a fixed value, such that additional flexibility is available when choosing which antennas and subcarriers to activate. Furthermore, two of the proposed algorithms perform bit loading in tandem per subcarrier to increase the overall system throughput, while satisfying a mean bit error rate (BER) constraint. As a result, the advantages offered by both frequency and spatial diversity are exploited.

The rest of this paper is organized as follows: Section 2 provides some details on the implementation of our multicarrier system that employs multiple antennas at both the transmitter and the receiver. The proposed algorithms are described in Section 3 while the simulation results of these algorithms are presented in Section 4. Section 5 contains several concluding remarks and final observations.

\section{System Implementation}

A schematic of the multicarrier transceiver employing multiple antennas is shown in Fig. 1. Referring to Fig. 1(a), the high-speed data stream $x(n)$ is demultiplexed into $N$ parallel data streams of different rates and modulated into $N$ streams 


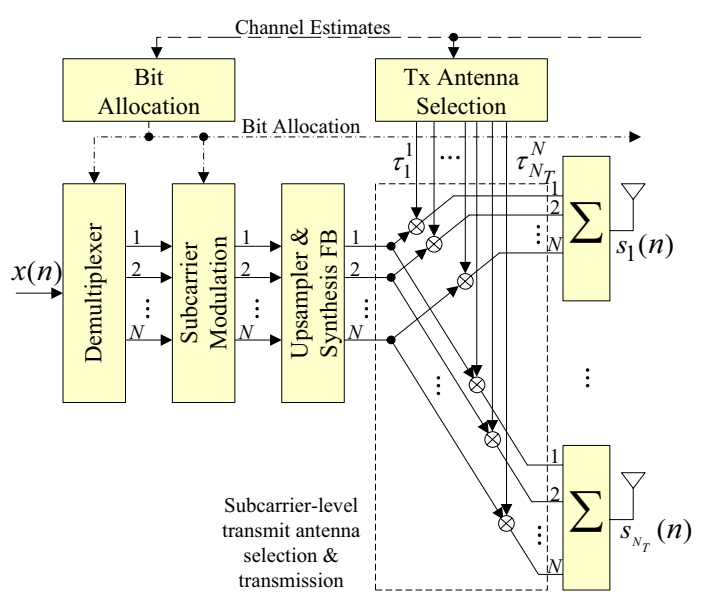

(a) Multicarrier transmitter with multiple antennas.

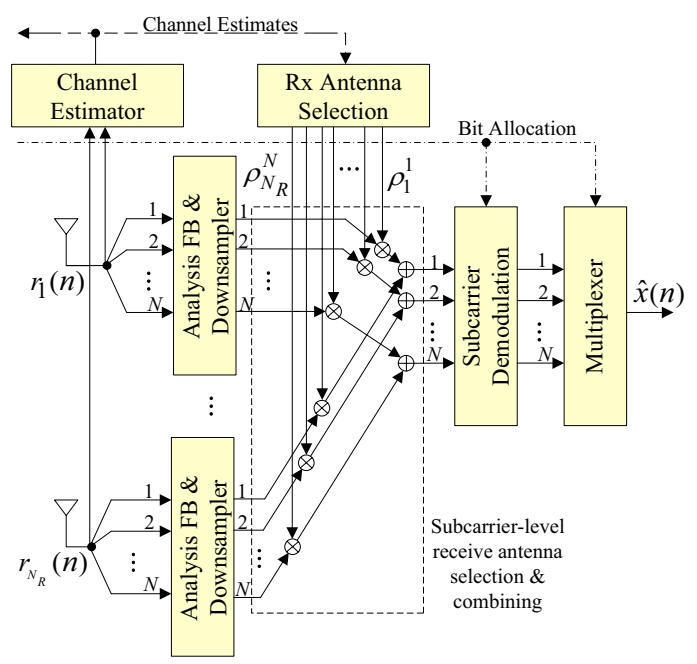

(b) Multicarrier receiver with multiple antennas.

Fig. 1 Schematics of the multicarrier transmitter and receiver employing multiple antennas in a diversity configuration.

of symbols. The choice of subcarrier modulation schemes and data rates are determined by the bit allocation algorithm employed by the system. These streams are then upsampled by a sampling factor $N$ and filtered by one of $N$ bandpass filters constituting the synthesis filterbank. The $N$ subcarrier outputs of the synthesis filterbank are fed to the $N_{T}$ antennas for transmission. However, the transmit antenna selection algorithm chooses which subset of antennas should transmit each subcarrier signal, turning off the corresponding subcarriers on the other antennas ${ }^{1}$. At each transmit antenna, the subcarriers are summed, forming the signals $s_{i}(n), i=1, \ldots, N_{T}$, and transmitted.

At the receiver, the $N_{R}$ receive antennas intercept the signals $r_{j}(n), j=1, \ldots, N_{R}$, as shown in Fig. 1(b). The analysis filterbanks at each receive antenna separates $r_{j}(n)$ into $N$ subcarriers and downsamples them by a sampling factor $N$. The

\footnotetext{
${ }^{1}$ The transmit antenna selection is an on/off procedure, where in Fig. 1(a), $\tau_{j}^{i}=0$ turns off subcarrier $i$ being fed into transmit antenna $j$, while $\tau_{j}^{i}=1$ leaves it on.
}

receive antenna selection algorithm then chooses which set of antennas to use for each subcarrier in the signal combining process $^{2}$. The combined subcarriers are then demodulated and multiplexed together, forming the reconstructed high-speed data stream $\hat{x}(n)$. Note that equalization would also be employed by the system at some stage in the receiver on each subcarrier to compensate for the distortion introduced to the signal $s_{i}(n)$ by the multiple input/multiple output (MIMO) channel. In the next section, the MIMO channel model employed in this work will be outlined.

\section{Proposed Algorithms}

The primary objective of the proposed algorithms is to increase the overall throughput of the system while ensuring the mean BER, $\bar{P}$, is below a specified limit, $P_{T}$. Note that this approach has been used in several single-input singleoutput (SISO) multicarrier bit loading algorithms [11]. To satisfy this objective, the algorithms are designed to search for the appropriate number of bits per symbol, $b_{i}$, and the transmit/receive antenna configuration, $s_{i}$, for subcarrier $i$, where:

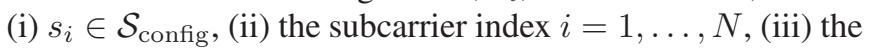
set $\mathcal{S}_{\text {config }}$ contains all possible transmit and receive antenna configurations, and (iv) $N$ is the total number of subcarriers. The secondary objective is to use as few antennas as possible to achieve the largest possible throughput. Thus, each subcarrier signal is broadcast simultaneously by a subset of transmit antennas rather than by all transmit antennas, as long as the throughput for each configuration is equivalent. Similarly, the intercepted copies of the subcarrier signals are picked up by a subset of, rather than all, the receive antennas as long as the throughput value of each configuration is equivalent.

Mathematically, the proposed algorithms attempt to solve a two-step optimization problem, where the first step is of the form:

$$
\max _{s_{i}, b_{i}} \sum_{i=1}^{N} b_{i}
$$

subject to :

$$
\bar{P}=\left(\sum_{i=1}^{N} b_{i} P_{i}\right) /\left(\sum_{i=1}^{N} b_{i}\right) \leq P_{T},
$$

where $P_{i}$ is the BER for subcarrier $i$, which is computed from the subcarrier signal-to-noise ratio (SNR), $\gamma_{i}$, via closed form expressions $[12]^{3}$. Now let $\mathcal{S}_{\max }$ denote the set of antenna configurations that yield the largest throughput in Eq. (1), where $\mathcal{S}_{\text {max }} \subseteq \mathcal{S}_{\text {config. }}$. The algorithms then solve the second step:

$$
\min _{s_{i} \in \mathcal{S}_{\max }}\left(\mu_{T, i} \cdot n_{T, i}\left(s_{i}\right)+\mu_{R, i} \cdot n_{R, i}\left(s_{i}\right)\right)
$$

\footnotetext{
${ }^{2}$ Referring to Fig. 1(b), the algorithm can either allocate a weight of $\rho_{j}^{i}=$ 0 to turn off subcarrier $i$ on receive antenna $j$, or a weight corresponding to either equal gain combining (EGC) or maximum ratio combining (MRC) of the $L_{R}$ antennas that are chosen for that subcarrier.

${ }^{3}$ The value of $\gamma_{i}$ is equal to the composite SNR value due to the recombining of the different signal paths from the transmitter to the receiver. Thus, $\gamma_{i}$ is also a function of the antenna subset configuration $s_{i}$.
} 
where $0<n_{T, i}\left(s_{i}\right) \leq N_{T}$ and $0<n_{R, i}\left(s_{i}\right) \leq N_{R}$ are the number of active transmit and receive antennas for antenna configuration $s_{i}, N_{T}$ and $N_{R}$ are the total number of transmit and receive antennas, and $\mu_{T, i} \& \mu_{R, i}$ are weights such that $\mu_{T, i}+\mu_{R, i}=1$. In this work, since minimizing the number of transmit and receive antennas is equally important, these weights are set to $\mu_{T, i}=\mu_{R, i}=0.5$.

The differences between the four proposed algorithms is due to the choice of additional constraints, namely,

$$
\begin{aligned}
& b_{1}=b_{2}=\cdots=b_{N}, \\
& s_{1}=s_{2}=\cdots=s_{N},
\end{aligned}
$$

where Eqs. (4) and (5) constrains the modulation schemes and the antenna subset configurations to be identical for all subcarriers. Therefore, the four proposed loading algorithms can be defined as:

- Signal-level antenna subset selection (i.e., employs both Eq. (4) and (5)),

- Subcarrier-level antenna subset selection (i.e., employs only Eq. (4)),

- Signal-level antenna subset selection with bit loading (i.e., employs only Eq. (5)),

- Subcarrier-level antenna subset selection with bit loading (i.e., employs neither constraint).

A detailed description of the proposed algorithm performing subcarrier-level antenna subset selection with bit loading is shown in Fig. 2. Note that the other three proposed algorithms have a similar structure, although with additional constraints. The theoretical values for $P_{i}$, given all possible antenna configurations and modulation schemes, are computed and stored in a look-up table for easy access. Then Steps 2 and 3 are performed to allow a quick exit in case it is not worth performing antenna subset selection and bit allocation. The algorithm then performs a search of each subcarrier and antenna configuration for the largest signal constellation such that $P_{i} \leq \hat{P}$ is satisfied, where $\hat{P}$ is the peak BER limit for any given subcarrier. The antenna configuration that yields the largest subcarrier throughput is then chosen for each subcarrier. In the case of a tie, the configuration employing the fewest antennas is chosen. Then the mean BER $\bar{P}$ is computed and the value of $\hat{P}$ is modified by an amount $\delta$. Steps 4 and 5 are then repeated with the new value of $\hat{P}$ to yield a new configuration, from which the mean BER $\bar{P}^{\prime}$ is computed. The values of $\bar{P}$ and $\bar{P}^{\prime}$ are compared with $P_{T}$ and their relation with the mean BER threshold will determine the next steps the algorithm will perform. If both are above or below $P_{T}$, the value of $\hat{P}$ is steadily modified until $\bar{P}$ and $\bar{P}^{\prime}$ straddle $P_{T}$, in which case the algorithm zooms in on $P_{T}$. The algorithm stops when it has found a configuration and allocation that has maximized the throughput while satisfying $\bar{P} \leq P_{T}$.

\section{Simulation Results}

To evaluate the performance of the proposed algorithms, a multicarrier system based on some of the operating parameters
1. Initialization: Compute $P_{i}, i=1, \ldots, N$, for all available modulation schemes and antenna configurations.

2. If the largest $P_{i}$ for the largest available signal constellation is less than $P_{T}$, set all subcarriers to that constellation, employ 1 transmit antenna, employ 1 receive antenna, and exit algorithm, else go to step 3.

3. If smallest $P_{i}$ for the smallest (non-zero) signal constellation is greater than $P_{T}$, null all subcarriers and exit algorithm, else proceed to step 4.

4. Find largest signal constellation for all subcarriers and antenna configurations such that $P_{i}<\hat{P}$, where $\hat{P}$ is the peak BER limit per subcarrier.

5. Select antenna configuration with largest $b_{i}$, $i=1, \ldots, N$ (in case of a tie in the number of bits, choose configuration with fewest overall antennas).

6. Compute $\bar{P}$ using Eq. (2).

7. If $\bar{P}<P_{T}$, let $\hat{P}=\hat{P}+\delta$, else $\hat{P}=\hat{P}-\delta$, where $\delta$ is the stepsize.

8. Find largest signal constellation for all subcarriers and antenna configurations such that $P_{i}<\hat{P}$.

9. Select antenna configuration with largest $b_{i}$, $i=1, \ldots, N$ (in case of a tie in the number of bits, choose configuration with fewest overall antennas).

10. Compute $\bar{P}^{\prime}$.

11. If both $\bar{P}>P_{T}$ and $\bar{P}^{\prime}>P_{T}$ (resp. $\bar{P} \leq$ $P_{T}$ and $\left.\bar{P}^{\prime} \leq P_{T}\right)$, and no previous straddling of $P_{T}$, let $\bar{P}=\bar{P}^{\prime}, \hat{P}=\hat{P}-\delta$ (resp. $\hat{P}=\hat{P}+\delta$ ), and go to step 8, else go to step 12 .

12. If both $\bar{P} \leq P_{T}$ and $\bar{P}^{\prime} \leq P_{T}$, and $P_{T}$ was straddled before, let $\bar{P}=\bar{P}^{\prime}, \hat{P}=\hat{P}+\delta$, and go to Step 8, else go to Step 13.

13. If both $\bar{P}$ and $\bar{P}^{\prime}$ are straddling $P_{T}$ and the number of times this occurred is less than a specified amount, reduce $\delta$, let $\bar{P}=\min \left(\bar{P}, \bar{P}^{\prime}\right)$, set $\hat{P}=\hat{P} \pm \delta$ (the future $\bar{P}^{\prime}$ should be on the same side of $P_{T}$ as $\bar{P}$ ), and go to step 8 . Otherwise, finalize the allocation and end the algorithm.

Fig. 2 Proposed subcarrier-level antenna subset selection algorithm with bit loading.

used in the IEEE 802.11a standard are employed $[13]^{4}$. The option to null subcarriers also exists in circumstances where the prevailing channel conditions are too poor. However, unlike the standard, where the same modulation scheme is employed across all the subcarriers, the proposed algorithms that employ bit loading can use a different modulation scheme for each subcarrier. Results from all the proposed algorithms were obtained for a target BER value of $P_{T}=10^{-5}$.

The antenna elements employed by the arrays are $\lambda / 2$ omnidirectional dipole antennas ${ }^{5}$ placed in a uniformly-spaced linear array with adjacent antenna separation of $d$ and oriented such that they are all perpendicular to the $x y$-plane, i.e., vertically polarized. For physical space considerations, the largest

\footnotetext{
${ }^{4}$ The system possesses $N=64$ subcarriers (with 6 "guard subcarriers" at each end of the 16.6 MHz bandwidth), uses BPSK, QPSK, square 16-QAM, and square 64-QAM modulation, and has an operating frequency of $5 \mathrm{GHz}$.

${ }^{5}$ The wavelength is equal to $\lambda=1 /(5 \mathrm{GHz})=0.06 \mathrm{~m}$.
} 


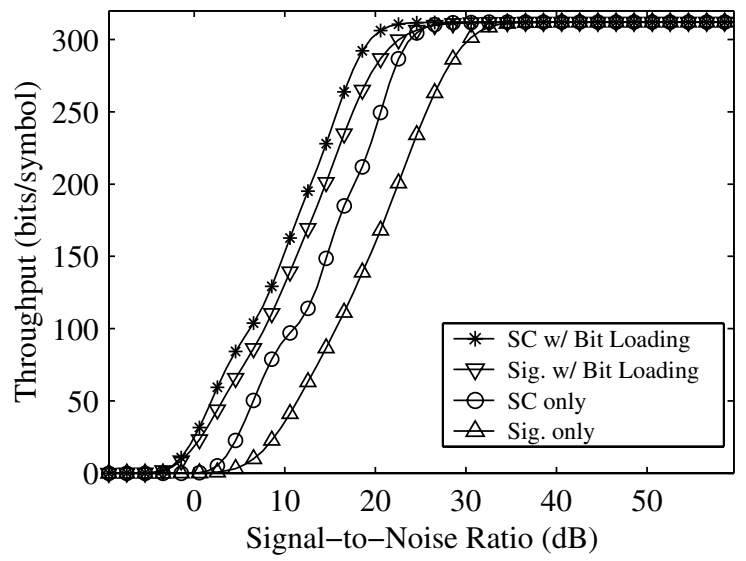

Fig. 3 Throughput results for different algorithms employed by a $\left(N_{T}=2, N_{R}=2\right)$ multicarrier system. Note that "SC" and "Sig." stand for subcarrier-level and signal-level antenna subset selection algorithms.

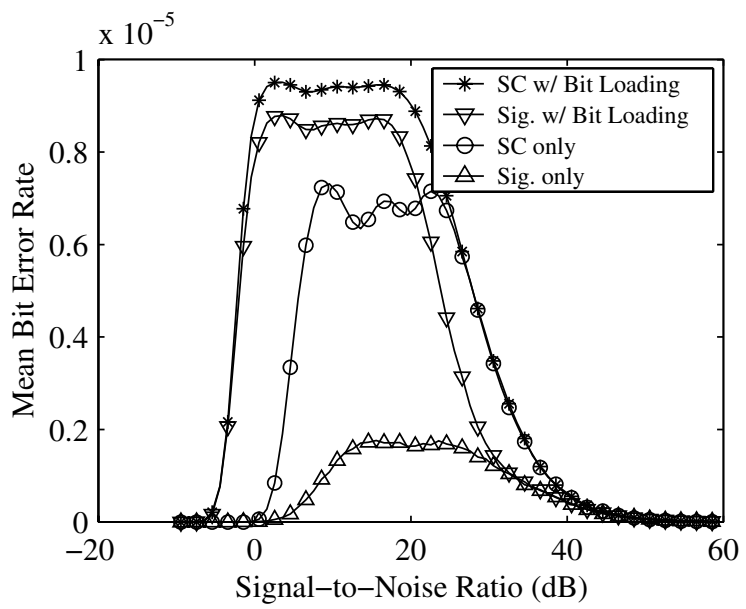

Fig. 4 Mean BER results for different algorithms employed by a $\left(N_{T}=2, N_{R}=2\right)$ multicarrier system. Note that "SC" and "Sig." stand for subcarrier-level and signal-level antenna subset selection algorithms.

array configuration employed at either the transmitter or receiver is 2 antennas ${ }^{6}$. Simple transmit diversity is employed at the transmitter while maximal ratio combining (MRC) is performed at the receiver to recombine the received signals ${ }^{7}$. The physical separation between the transmitter and receiver was varied between $1 \mathrm{~m}$ and $60 \mathrm{~m}^{8}$. The MIMO channel consists of a collection of SISO channel responses generated using the method proposed by Saleh and Valenzuela $[14]^{9}$. The SISO components of the MIMO channel were assumed to be uncorrelated since the adjacent antenna separation was set to

\footnotetext{
${ }^{6}$ Although more antennas would improve system performance, this enhancement would come at the cost of increased computational complexity for the algorithm.

${ }^{7}$ Results for when equal gain combining (EGC) is employed showed a small decrease in throughput performance.

${ }^{8}$ The change in transmitter/receiver separation distance corresponds to an SNR change ranging from $59 \mathrm{~dB}$ to $-11 \mathrm{~dB}$.

${ }^{9}$ The SISO components were assumed to be time-invariant, non-line-ofsight, and uncorrelated.
}

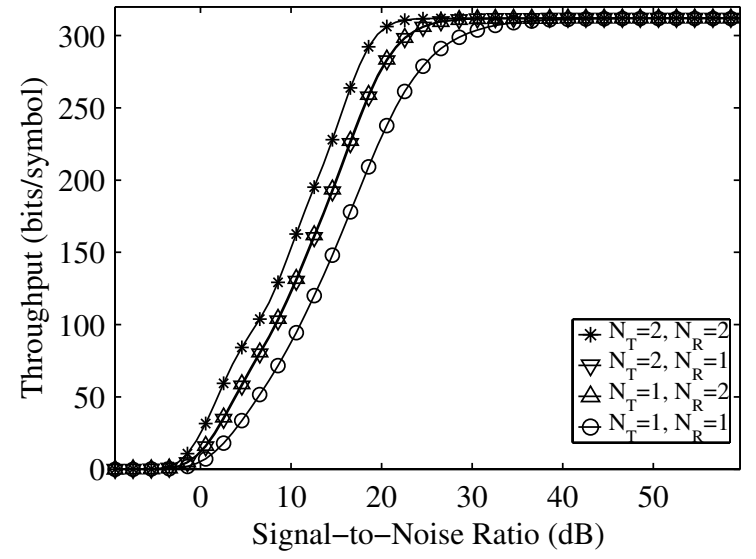

Fig. 5 Throughput results for different array configurations using the proposed antenna subset selection algorithm (subcarrier-level) with bit loading.

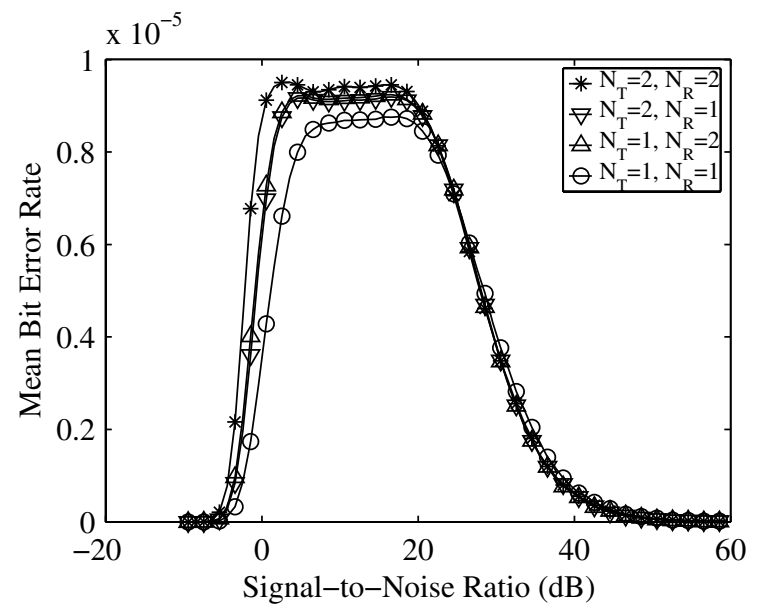

Fig. 6 Mean BER results for different array configurations using the proposed antenna subset selection algorithm (subcarrier-level) with bit loading.

$d=5 \lambda$. However, the effect of antenna separation on system performance was also examined for $d=\lambda$ and $d=0.25 \lambda$ using the model proposed in [15]. Finally, for each MIMO channel realization, the algorithms were operating at 70 different averaged SNR values equally spaced in the logarithmic domain. The trials were repeated for 10000 different MIMO channel realizations and the results averaged.

Referring to Figs. 3 and 4, the throughput and mean BER results of the proposed algorithm and its three variants are presented for an $\left(N_{T}=2, N_{R}=2\right)$ multicarrier system. It can be observed that the proposed algorithm possesses the largest throughput values, followed by the signal-level antenna subset selection algorithm with bit loading, the subcarrier-level antenna subset selection algorithm, and the signal-level antenna subset selection algorithm. For example, at an SNR of $20 \mathrm{~dB}$, the throughput of a $(2,2)$ system employing these four algorithms are 305, 280, 240, and 150 bits per symbol, respectively. This is due to the increased number of available configurations that the algorithms can choose, with the proposed algorithm having the most flexibility. This is evident from the 


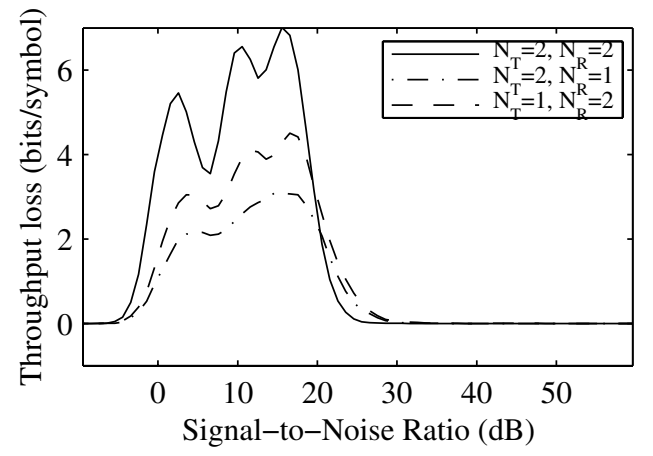

Fig. 7 Throughput loss due to correlation for the subcarrier-level antenna subset selection algorithm employing bit loading when $d=$ $\lambda$.

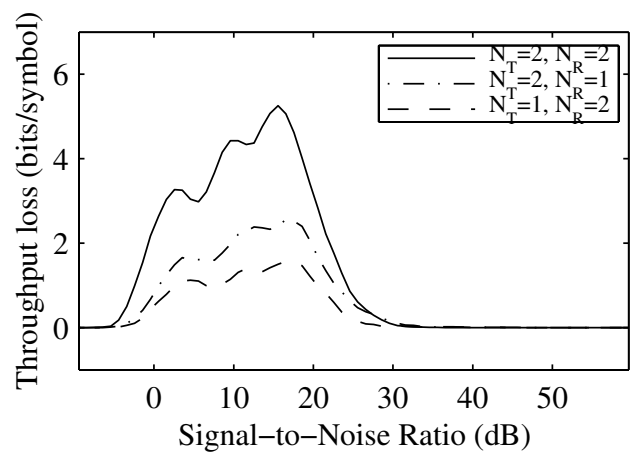

Fig. 8 Throughput loss due to correlation for the signal-level antenna subset selection algorithm employing bit loading when $d=\lambda$.

mean BER results shown in Fig. 4, where the proposed algorithm comes the closest to $P_{T}=10^{-5}$ while the others are farther away from $P_{T}$. Another observation is that as the total number of antennas employed by the system increases, so does the throughput, as shown in Fig. 5 for the proposed algorithm. This is due to the system further exploiting the spatial diversity offered by these arrays. Moreover, more configurations are available to the algorithms, thus given them more flexibility in reaching a larger throughput, evident from the mean BER results in Fig. 6.

As for the effects of correlation, the system experiences a very small decrease in throughput for $d=\lambda$, as shown in Figs. 7 and 8. Simulations have shown that for $d=0.25 \lambda$, the system experiences a loss of at most 30 bits per symbol. However, even with the loss in throughput for $d=0.25 \lambda$, the simulation results show that it is advantageous to use MIMO and bit loading.

\section{Conclusion}

A novel algorithm was proposed that performs both antenna subset selection and bit loading in tandem for a multicarrier system employing multiple antennas. The proposed algorithm, as well as three simpler variants, can increase the overall throughput of the system while being constrained by a mean BER limit. The results showed that the proposed algorithm achieved the best throughput performance at the cost of the largest computational complexity. When correlation in the MIMO channel model is considered, the algorithms show only a negligible throughput degradation for $d=\lambda$ and a small decrease for $d=0.25 \lambda$. Thus, combining antenna subset selection and bit loading offers substantial gains in terms of increased throughput.

\section{References}

[1] A. J. Paulraj, D. A. Gore, R. U. Nabar, and H. Bolcskei, "An overview of MIMO communications - A key to gigabit wireless," Proc. IEEE, vol. 92, no. 2, pp. 198-218, Feb. 2004.

[2] A. F. Molisch and M. Z. Win, "MIMO systems with antenna selection," IEEE Microwave Mag., pp. 46-56, Mar. 2004.

[3] R. S. Blum, "MIMO capacity with antenna selection and interference," in Proc. IEEE Int. Conf. Acoust., Speech, Signal Process., Hong Kong, China, Apr. 2003, vol. 4, pp. 824-827.

[4] A. Gorokhov, "Transmit diversity versus SDMA: Analytic and numerical comparisons," in Proc. IEEE Int. Conf. Commun., New Orleans, LA, USA, June 2000, vol. 2, pp. 1020-1024.

[5] Y. G. Li, J. C. Chuang, and N. R. Sollenberger, "Transmitter diversity for OFDM systems and its impact on high-rate data wireless networks," IEEE J. Select. Areas Commun., vol. 17, no. 7, pp. 1233-1243, July 1999.

[6] S. Thoen, L. Van de Perre, B. Gyselinckx, and M. Engels, "Performance analysis of combined transmit-SC/receive-MRC," IEEE Trans. Coтmun., vol. 49, no. 1, pp. 5-8, Jan. 2001.

[7] J. Rinne, "Subcarrier-based selection diversity reception of DVB-T in a mobile environment," in Proc. 50th IEEE Veh. Technol. Conf. - Fall, Amsterdam, Netherlands, Sept. 1999, vol. 2, pp. 1043-1047.

[8] X. Ouyang, M. Ghosh, and J. P. Meehan, "Optimal antenna diversity combining for IEEE 802.11a system," IEEE Trans. Consumer Electron., vol. 48, no. 3, pp. 738-742, Aug. 2002.

[9] H. Shi, M. Katayama, T. Yamazato, H. Okada, and A. Ogawa, "An adaptive antenna selection scheme for transmit diversity in OFDM systems," in Proc. 54th IEEE Veh. Technol. Conf. - Fall, Atlantic City, NJ, USA, Oct. 2001, vol. 4, pp. 2168-2172.

[10] D. Lee, G. J. Saulnier, Z. Ye, and M. J. Medley, "Antenna diversity for an OFDM system in a fading channel," in Proc. IEEE Military Commun. Conf., Atlantic City, NJ, USA, Oct. 1999, vol. 2, pp. 11041109.

[11] A. M. Wyglinski, F. Labeau, and P. Kabal, "An efficient bit allocation algorithm for multicarrier modulation," in Proc. IEEE Wireless Commun. Networking Conf., Atlanta, GA, USA, Mar. 2004, vol. 2, pp. 1194-1199.

[12] J. G. Proakis, Digital Communications, McGraw-Hill, New York, NY, USA, 3rd edition, 1995.

[13] Institute of Electronics and Electrical Engineers, "Wireless LAN medium access control (MAC) and physical layer (PHY) specifications: High-speed physical layer in the $5 \mathrm{GHz}$ band," IEEE Standard 802.11a, Nov. 1999.

[14] A. A. M. Saleh and R. A. Valenzuela, "A statistical model for indoor multipath propagation," IEEE J. Select. Areas Commun., vol. 5, no. 2, pp. 128-137, Feb. 1987.

[15] D. Gesbert, H. Bolcskei, D. A. Gore, and A. J. Paulraj, "Outdoor MIMO wireless channels: Models and performance prediction," IEEE Trans. Commun., vol. 50, no. 12, pp. 1926-1934, Dec. 2001. 